\title{
EDITORIAL
}

\section{Pregnancy Immunology}

The diverse papers addressing immune responses, specific and nonspecific, within the local uterine environment, as well as in extra-uterine sites, presented in this session of the Third Annual International Symposium on the Immunology of Reproduction were based on studies conducted utilizing those techniques which gained standard usage in the decade 1970 to 1980.

In this section, investigators presented the results of studies which examined cellular and humoral immune mechanisms operating in the local uterine environment; alterations in systemic maternal immunoreactivity, both that which is specific to the conceptus and that which is nonspecific; and activities of sets and subsets of immunoreactive cells studied both before and during pregnancy. In the animal studies, local immunoreactivity within the uterus was assessed. M. Kearns and P. Lala (Am J Reprod Immunol issue 3:2) claim that uterine decidual cells in pseudopregnant mice were of bone marrow origin. They produced bone marrow chimeras by repopulating lethally irradiated Fl hybrid mice and identified decidual cells bearing donor-specific antigens utilizing an immunolabeling technique with monospecific anti- $\mathrm{H} 2$ antibodies. This interesting finding requires confirmation; however, the possibility that decidual cells have immune functions necessary for the maintenance of pregnancy is exciting. J.P. Krcek and his associates studied the accumulation of mononuclear cells in the vicinity of the trophoblastic giant cells in the pregnant mouse uterus. The infiltrate was more pronounced on the tenth day of pregnancy in matings with $\mathrm{H} 2$ haplotype differences. The fact that the mononuclear leukocyte accumulation was localized to the implantation site supports the concept of immunological recognition on an antigen-specific basis R.N. Smith and his associates analyzed the maternal alloantibody response in allogenic pregnancies in rats. The primary and secondary alloantibody response following pregnancy were quite different from alloantibody responses following conventional alloimmunization. During pregnancy, the predominant source of the alloantibody was found to be in the spleen and was specific towards the conceptus. D.A. Clark and R.M. Slapsys (Am J Reprod Immunol issue $3: 2$ ) previously documented the presence of suppressor cells in lymph nodes draining the uterus of allogenically mated mice. Further studies showed that these cells appeared first in the uterine decidua after mating and reached their maximum concentration at the time of implantation. Their suppressive activity was not specific for the paternal MHC antigens but selectively inhibited the generation of cytotoxic $\mathrm{T}$ lymphocytes.

Address reprint requests to Alan E. Beer. M.D.. Department of Obstetrics and Gynecology, University of Michigan School of Medicine, Ann Arbor, MI 48109.
Three interesting papers on natural killer (NK) cell activity during pregnancy were in agreement that there was a decrease in NK activity during pregnancy and that the activity could be enhanced or augmented by interferon. The significance of this depressed natural killer activity awaits definition as to its role in normal or abnormal pregnancy. The interesting observation of C. Coulam and her associates showing marked increase in the major basic protein of eosinophil graunules during pregnancy and the disappearance of these eosinophilic granules in the postpartum period raises the question of the role, if any, of this protein in disorders of pregnancy. R.M. Galbraith and his associates (Am J Reprod Immunol issue 3:1) utilizing immunohistochemical techniques, claimed activated lymphocytes express an isoenzyme of placental alkaline phosphatase. Antisera prepared to placental alkaline phosphatase were efficient in blocking both alloantigen and mitogen responses in culture, indicating that this cell membrane component is essential for these immunological reactions. It is possible that the placental alkaline phosphatase demonstrated on activated lymphocytes and placental trophoblast may be the trophoblast lymphocyte cross-reacting antigen TLX, described by W.P. Faulk et al, and is consistent with his demonstration of the immunosuppressive properties of the anti-TLX antisera. Redman and his associates (Am J Reprod Immunol issue $3: 4$ ) studied maternal cell-mediated immunity to paternal HLA antigens in both normal and preeclamptic pregnancy, using mixed lymphocyte culture responses and measuring blast transformation and the production of leukocyte migration inhibitory factor (LIF) in parallel. They found that the responses in preeclamptic couples were consistently and significantly different from those of normal couples in that the paternal response to the maternal lymphocytes in one-way reactions was significantly reduced. This was shown not to be a function of the HLA type of the individuals.

The next ten years promise to be exciting for reproductive immunology. New methods are becoming available which enable us to look at immunoreactivity in more precise ways at all phases of human and animal pregnancy. Application of the new insights of molecular biology should ensure a clearer understanding of the privileged immunological status of the fetal trophoblast and the elucidation of disorders of reproduction which have an immune basis.

\section{Alan E. Beer \\ University of Michigan Ann Arbor}

Chris W.G. Redman

University of Oxford

England 